\title{
Moniteur individuel de la contamination atmosphérique alpha (Type Monica $\alpha)^{1}$
}

(Manuscrit reçu le 24 octobre 1983)

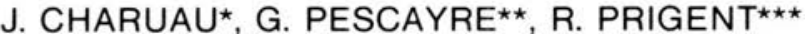

\section{RÉSUME}

L'étude d'un détecteur individuel de la contamination atmosphérique alpha consiste essentiellement à définir la forme et les dimensions de la tête de prélèvement, ainsi que le type de filtre a utiliser. L'appareil doit :

- aspirer les aérosols avec un rendement de captation voisin de $100 \%$ jusqu'au diamètre aérodynamique (densité 1) de $10 \mu \mathrm{m}$;

- conduire les plus grosses particules aspirées jusqu'au filtre de prélèvement sans perte importante sur les parois ;

- répartir ces particules de manière homogène sur le filtre après séparation des aérosols radioactifs naturels descendants du Rn et du Th ;

- mesurer avec le meilleur rendement possible l'activité $\alpha$ déposée ;

- délivrer une alarme sonore sous forme d'impulsions dont la fréquence varie avec l'activité volumique de l'air.

\section{ABSTRACT}

The development of an individual monitor of $\alpha$ atmospheric contamination must take into account the shape and size of the sampling head and the filter type. The monitor should :

- draw in the aerosols up to an aerodynamic diameter (density 1) of $10 \mu \mathrm{m}$ with a recovery efficiency near $100 \%$;

- lead the biggest particles up to the sampling filter without any signicant loss on the walls ;

- after separation of natural radioactive aerosols (Rn and Th daughters) distribute the particles on the filter uniformly ;

- measure the $\alpha$ activity deposited with the best efficiency ;

- deliver a sound alarm as impulsions with frequencies varying with the volumic activity of the atmosphere.

1. Communication présentée aux journées d'études "Plutonium et radioprotection", Saclay, 14-16 juin 1983.

* Commissariat à l'énergie atomique, IPSN, Département de protection technique, DPT/SPIN, 91191 Gif-sur-Yvette Cedex.

** Commissariat à l'énergie atomique, Service de protection contre les rayonnements, Centre de Valduc, BP 14, 21120 Is-sur-Tille.

$\star \star \star$ Commissariat à l'énergie atomique, IPSN, Département de protection technique (DPT/SIDR), BP 6, 92260 Fontenay-aux-Roses. 


\section{POURQUOI UN MONITEUR INDIVIDUEL DE LA CONTAMINATION ATMOSPHERIQUE ALPHA}

La surveillance individuelle des travailleurs dans le cas de l'irradiation externe est suivie officiellement par le film dosimètre. Cependant, pour éviter des dépassements éventuels des limites autorisées, les travailleurs peuvent porter différents dosimètres à lecture directe (stylodosimètre, dosimètre à alarme) car l'information donnée par le film n'est connue qu'après un ou deux mois. D'autre part, ils sont aussi surveillés par des systèmes de balises de zone qui les avertissent lorsque les conditions de travail ont changé.

Dans le cas de la contamination, les travailleurs sont protégés et informés bien sûr par des balises de zone et sont soumis à une surveillance médicale spécifique pour déterminer le cas échéant l'équivalent de dose engagée due à l'irradiation interne par inhalation. Ils peuvent porter aussi des appareils de prélèvements individuels d'aérosols qui sont à lecture différée, mais il n'existe pas de système à lecture directe.

Si l'on admet le port d'un dosimètre à alarme dans le cas de l'irradiation externe, l'on peut admettre le port d'un appareil individuel de prélèvement d'aérosols radioactifs à information directe pour la contamination. L'on peut dire que cet appareil doit être à la contamination ce qu'est le dosimètre à alarme pour l'irradiation.

Des réflexions et des études menées au sein du groupe de travail GTR 4 du comité d'instrumentation de radioprotection du Commissariat à l'énergie atomique ont montré qu'il y avait d'autres éléments qui plaidaient en faveur du développement d'un tel appareil :

- Les risques de fuite (perte de confinement) se situent avec une grande probabilité près des opérateurs (cas des opérations en boîte à gants) d'où l'intérêt de donner l'alarme au plus près de la source.

- Le filtre du moniteur peut être exploité ultérieurement si besoin est.

- Une étude du transfert des gaz et des aérosols dans les ateliers où l'on travaille sur le plutonium a montré que la vitesse de transfert horizontal pouvait suivant les cas être comprise entre 2 et $16 \mathrm{~cm} . \mathrm{s}^{-1}$. Dans ce cas, une bouffée de contamination ne pourra atteindre le détecteur de zone qu'après un temps de $200 \mathrm{~s}$ si la vitesse de transfert est de $5 \mathrm{~cm}$. $\mathrm{s}^{-1}$ et la distance entre le lieu d'émission et l'emplacement de la balise est de $10 \mathrm{~m}$.

- Cette étude a montré aussi que les coefficients de transfert $^{2}$ (proportionnels à la concentration des aérosols) pouvaient être très différents entre le lieu d'émission et divers emplacements se situant dans l'atelier. C'est le cas de la bouffée de contamination émise près du travailleur où le coefficient de transfert peut être de 10 à 20 fois supérieur à celui trouvé en divers emplacements, ce qui améliore la sensibilité relative du prélèvement individuel par rapport à la balise de zone.

- L'appareil individuel peut avoir un effet psychologique certain sur les opérateurs, il peut être sécurisant.

2. Le coefficient de transfert $\left(\mathrm{s}_{\mathrm{m}} \mathrm{m}^{-3}\right)$ est égal au rapport de la concentration massique de l'aérosol en un point $\left(\mathrm{kg} \cdot \mathrm{m}^{-3}\right)$ au flux d'émission massique de la source $\left(\mathrm{kg} \cdot \mathrm{s}^{-1}\right)$. 
Le prélèvement individuel à information directe nous semble être complémentaire d'une balise de zone à grande sensibilité judicieusement placée, et mériter un développement industriel.

\section{CONCEPTION ET EXPERIMENTATION DE LA TETE DE PRELEVEMENT}

\section{Objectif}

L'étude d'un détecteur individuel de la contamination atmosphérique alpha consiste essentiellement à définir la forme et les dimensions de la tête de prélèvement, ainsi que le type de filtre à utiliser. L'appareil doit :

- aspirer les aérosols avec un rendement de captation voisin de 100 \% jusqu'au diamètre aérodynamique (densité 1) de $10 \mu \mathrm{m}$;

- conduire les plus grosses particules aspirées jusqu'au filtre de prélèvement sans perte importante sur les parois ;

- répartir ces particules de manière homogène sur le filtre ;

- mesurer avec le meilleur rendement possible l'activité $\alpha$ déposée ;

- délivrer une alarme sonore sous forme d'impulsions dont la fréquence varie avec l'activité volumique de l'air.

L'appareil étant destiné à prévenir l'individu d'une contamination atmosphérique par des émetteurs $\alpha$, principalement les transuraniens, les produits de filiation solides du radon peuvent en atténuer la sensibilité de mesure. Compte tenu des dimensions généralement supérieures au micromètre des contaminants, et inférieures au micromètre des descendants du radon, l'étude vise à effectuer la séparation dichotomique des aérosols et à éliminer les particules submicroniques.

Nous avons effectué des mesures granulométriques à l'aide d'impacteurs multi-étages sur des poudres de $\mathrm{PuO}_{2}$ dans des boîtes à gants de l'usine Cogéma de La Hague, ainsi que sur des mélanges $\left(\mathrm{UO}_{2}+\mathrm{PuO}_{2}\right)$ lors d'incidents de contamination aux ateliers plutonium du CEN-Cadarache. Le diamètre aérodynamique moyen en activité est compris entre 2 et $6 \mu \mathrm{m}$.

\section{Conception}

\subsection{Conception générale}

L'appareil doit entraîner un minimum d'inconvénients à son porteur, et notamment en ce qui concerne son encombrement, son poids (batterie), et le bruit de sa pompe. Aussi le débit d'aspiration est-il limité à 3 I. $\mathrm{min}^{-1}$, débit des prélèvements individuels portés au CEA. La mesure est faite par une diode à barrière de surface dont le diamètre sensible est de $24 \mathrm{~mm}$; on choisit de la placer, avec l'électronique associée, dans un volume sphérique ; l'écoulement de l'air et des aérosols se fait entre cette sphère et une enveloppe concentrique (fig. 1). Afin de minimiser la précipitation électrostatique des particules chargées sur les parois, celles-ci sont métalliques ou métallisées. Le filtre (diamètre d'aspiration : $21 \mathrm{~mm}$ ) doit être le plus près possible de la surface sensible du détecteur. Cet ensemble sphérique (diamètre extérieur : $55 \mathrm{~mm}$ ) consti- 
tue la tête de prélèvement; elle est montée sur un boîtier parallélépipédique en matière plastique. Celui-ci renferme un filtre absolu pour protéger la pompe se trouvant en aval, le haut-parleur miniaturisé fournissant l'alarme sonore, et la batterie. L'appareil ressemble à un boîtier d'éclairage électrique.

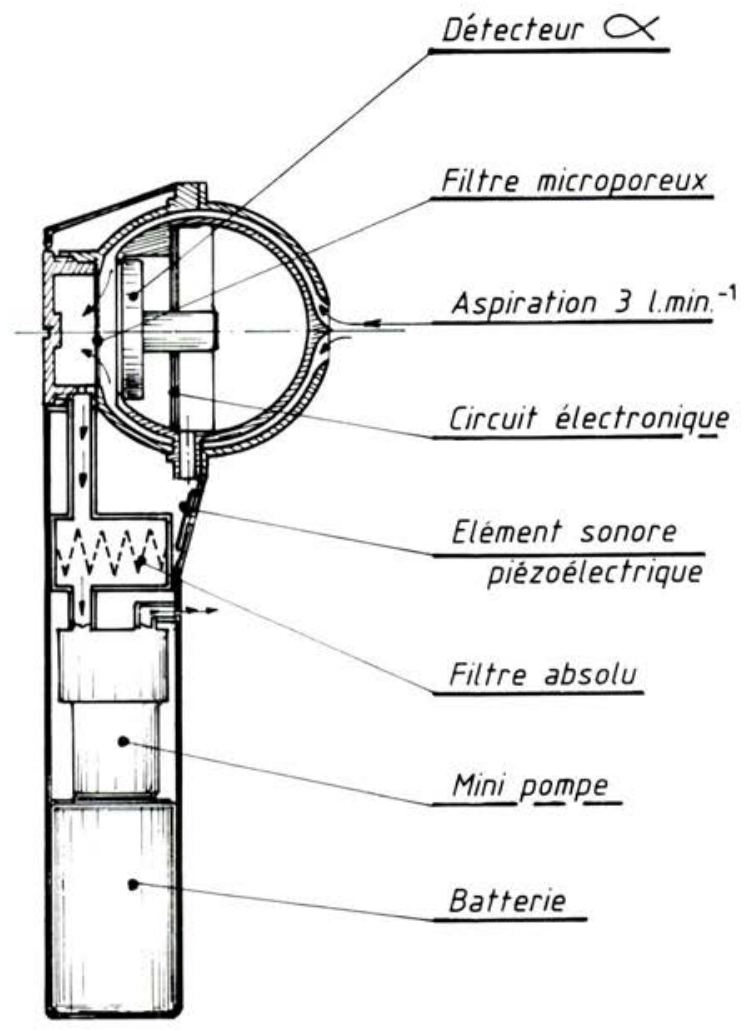

Fig. 1. - Schéma de l'appareil

\subsection{Orifice d'aspiration}

II est calculé à partir des critères de C.N. DAVIES [5] qui permettent l'échantillonnage des aérosols en air calme, avec un rendement de $100 \pm 4 \%$. Le rendement de captation est :

$$
\mathrm{n}=\frac{\mathrm{C}}{\mathrm{C}_{\mathrm{o}}}
$$

où $\mathrm{C}_{\text {。 }}$ est la concentration volumique vraie de l'aérosol (g. $\mathrm{cm}^{-3}$ d'air), et $\mathrm{C}$ est la concentration volumique aspirée par l'appareil. 
L'air est calme si sa vitesse $V$ est telle que $V \leqslant \frac{U_{0}}{5}$, où $U_{\circ}$ est la vitesse dynamique du prélèvement définie par:

$$
U_{0}=\left(\frac{Q}{4 \pi \cdot \tau^{2}}\right)^{1 / 3}
$$

où $Q$ est le débit de prélèvement ( $\left.3 \mathrm{I} \cdot \mathrm{min}^{-1}\right)$, et $\tau$ est le temps de relaxation des particules ; rappelons que :

$$
\tau=\frac{\mathrm{d}^{2} \mathrm{p} \cdot \rho_{\mathrm{p}}}{18 \mu} \cdot \mathrm{C}_{\mathrm{n}}
$$

si $d_{0}$ et $\rho_{0}$ sont le diamètre et la masse volumique des particules, $\mu$ est la viscosité dynamique de l'air, $\mathrm{C}_{\mathrm{n}}$ est le coefficient correctif de Cunningham $\left(\simeq 1\right.$ pour $\left.\mathrm{d}_{\mathrm{p}}>1 \mu \mathrm{m}\right)$.

Pour des particules de diamètre aérodynamique $10 \mu \mathrm{m}\left(\tau=3.10^{-4} \mathrm{~s}\right)$, il vient : $U_{0}=3,4 \mathrm{~m} \cdot \mathrm{s}^{-1}$, ce qui implique pour l'air calme $\mathrm{V} \leqslant 0,68 \mathrm{~m} \cdot \mathrm{s}^{-1}$.

Notons que nous avons mesuré dans différents laboratoires ventilés (A.T.Pu de Cadarache, LECl de Saclay, bâtiments 11 et 118 de Valduc) des vitesses de transfert de la contamination variant de 0,02 à $0,16 \mathrm{~m} \cdot \mathrm{s}^{-1}$ [3].

La comparaison des rendements de captation d'une fente ou d'un orifice circulaire est, dans notre cas, favorable à l'orifice tel que représenté en figure 1 . Son diamètre $D=12 \mathrm{~mm}$ respecte les conditions :

$$
10\left(\frac{Q \cdot \tau}{4 \pi}\right)^{1 / 3} \leqslant D \leqslant \frac{2}{5}\left(\frac{Q}{\pi \cdot g \cdot \tau}\right)^{1 / 2}
$$

où g est l'accélération de la pesanteur. Le calcul donne :

$$
10,6 \leqslant D \leqslant 29,1 \mathrm{~mm}
$$

\subsection{Profil aéraulique de la tête}

II s'agit essentiellement de calculer l'épaisseur e de la lame d'air comprise entre les deux sphères concentriques, de même que les rayons de courbure de la pointe du bulbe et de la chambre inférieure avant l'arrivée sur le filtre, afin de réduire le plus possible la perte des grosses particules sur les parois. Pour cela, l'on recherche:

- un écoulement laminaire, ou peu turbulent: nombre de Reynolds $R e<2500$, de manière à éviter le dépôt par diffusion turbulente ;

- des vitesses de dérive des particules, dues à la sédimentation $\left(v_{s}\right)$ ou à la force centrifuge $\left(v_{c}\right)$, qui soient faibles par rapport à la vitesse moyenne de l'écoulement $\bar{U}$, si possible inférieures à $1 \%$. Puisque :

$$
\begin{aligned}
& \mathrm{v}_{\mathrm{s}}=\mathrm{g} \cdot \tau \text { et } \mathrm{v}_{\mathrm{c}}=\frac{\tau \cdot \overline{\mathrm{U}}^{2}}{\mathrm{R}} \text { (où } R \text { est le rayon de courbure), il vient: } \\
& \frac{\overline{\mathrm{U}}}{\mathrm{v}_{\mathrm{s}}}=\frac{\overline{\mathrm{U}}}{\mathrm{g} \cdot \tau} \geqslant 100 \text { et } \frac{\bar{U}}{\mathrm{v}_{\mathrm{c}}}=\frac{\mathrm{R}}{\tau \cdot \overline{\mathrm{U}}} \geqslant 100 ;
\end{aligned}
$$

- une inertie relativement faible des grosses particules à l'entrée de l'appareil pour éviter leur impaction sur le bulbe : nombre de Stokes $<3.10^{-2}$.

Les données relatives à ce problème de dépôt sont extraites de [4]. L'optimisation aboutit aux dimensions suivantes : 
- diamètre moyen de la lame d'air (entre sphères) : $50 \mathrm{~mm}$;

- épaisseur de la lame d'air : 1,5 mm ;

- rayon de courbure du sommet du bulbe : $5 \mathrm{~mm}$;

- rayon de courbure de la chambre inférieure : $13 \mathrm{~mm}$;

- distance entre le filtre et la surface sensible du détecteur : $3 \mathrm{~mm}$.

\subsection{Piégeage de la fraction libre du radon}

Les particules ultrafines des descendants solides du radon non fixées sur les poussières atmosphériques constituent la fraction libre. Ce sont des particules électriquement chargées (gros ions) ou neutres, dont les diamètres sont compris entre $6.10^{-4}$ et $2.10^{-3} \mu \mathrm{m}$. Un pourcentage important de l'activité totale due au radon est sous cette forme; dans une mine d'uranium laboratoire, P. DUPORT [6] a mesuré $30 \%$ de RaA et $15 \%$ de $\mathrm{RaB}$ et $\mathrm{RaC}$; dans les laboratoires filtrés, il peut être supérieur.

Compte tenu de leur petite taille, la mobilité de ces particules est grande; elles diffusent rapidement et peuvent être piégées par les parois de la tête de prélèvement. Si l'on assimile le volume d'écoulement à un parallélépipède de même épaisseur $(0,15 \mathrm{~cm})$, de largeur I égale au parcours des particules dans la tête $(6 \mathrm{~cm})$, et de longueur telle que la surface des plaques soit égale à celle des parois de la tête, la vitesse $\bar{U}$ est équivalente. Selon GORMLEY et KENNEDY [6], la fraction pénétrante (ou transmise) est, pour un écoulement laminaire dans un canal rectangulaire :

$F_{p}=\frac{n}{n_{0}}=0,91 \exp (-1,885 \beta)+0,053 \exp (-21,43 \beta)$

si $\beta=\frac{D i . I}{h^{2} \cdot \bar{U}}>0,01$

où $n_{0}$ et $n$ sont les nombres de particules en amont et en aval du canal, $\mathrm{h}=\frac{1}{2}$ distance interplaques $=0,075 \mathrm{~cm}$,

et $\mathrm{Di}$ est le cœfficient moyen de diffusion des particules, soit $0,054 \mathrm{~cm}^{2} \cdot \mathrm{s}^{-1}$ [6]. On trouve $F_{\rho}=1,55 \cdot 10^{-2}$. La fraction retenue par les parois serait $98,5 \%$ de la fraction libre du radon.

La figure 2 montre que la fraction transmise varie de 0 à $100 \%$ lorsque le diamètre des particules croît de $10^{-3}$ à $2.10^{-2} \mu \mathrm{m}$ environ.

\subsection{Séparation dichotomique des aérosols par le filtre}

L'autre fraction des descendants solides du radon est fixée sur des particules naturelles formées par agglomération ou condensation à partir des particules ultra-fines (noyaux de condensation); le diamètre moyen de cet aérosol radioactif naturel est, selon les auteurs, compris entre 0,1 et $0,2 \mu \mathrm{m}$. Nous devons également chercher à l'éliminer [7].

Un moyen simple de séparer le spectre granulométrique, afin de ne garder que la fraction supérieure à $1 \mu \mathrm{m}$, est d'utiliser un filtre de prélèvement de type Nuclépore. II s'agit de membrane en polycarbonate d'épaisseur $10 \mu \mathrm{m}$, à pores capillaires. Comme l'ont montré D. BOULAUD et Coll. [1], l'efficacité de collection est grande pour les particules ultrafines (diffusion), puis passe par un minimum et croît de nouveau lorsque les 
particules ont un diamètre $d_{p}$ un peu plus petit que celui des pores $D$ : la collection s'effectue par impaction inertielle. Lorsque $\frac{d p}{D} \geqslant 1$, l'efficacité $E=100 \%$, par effet d'interception. La figure 3 montre cette variation d'efficacité de collection.

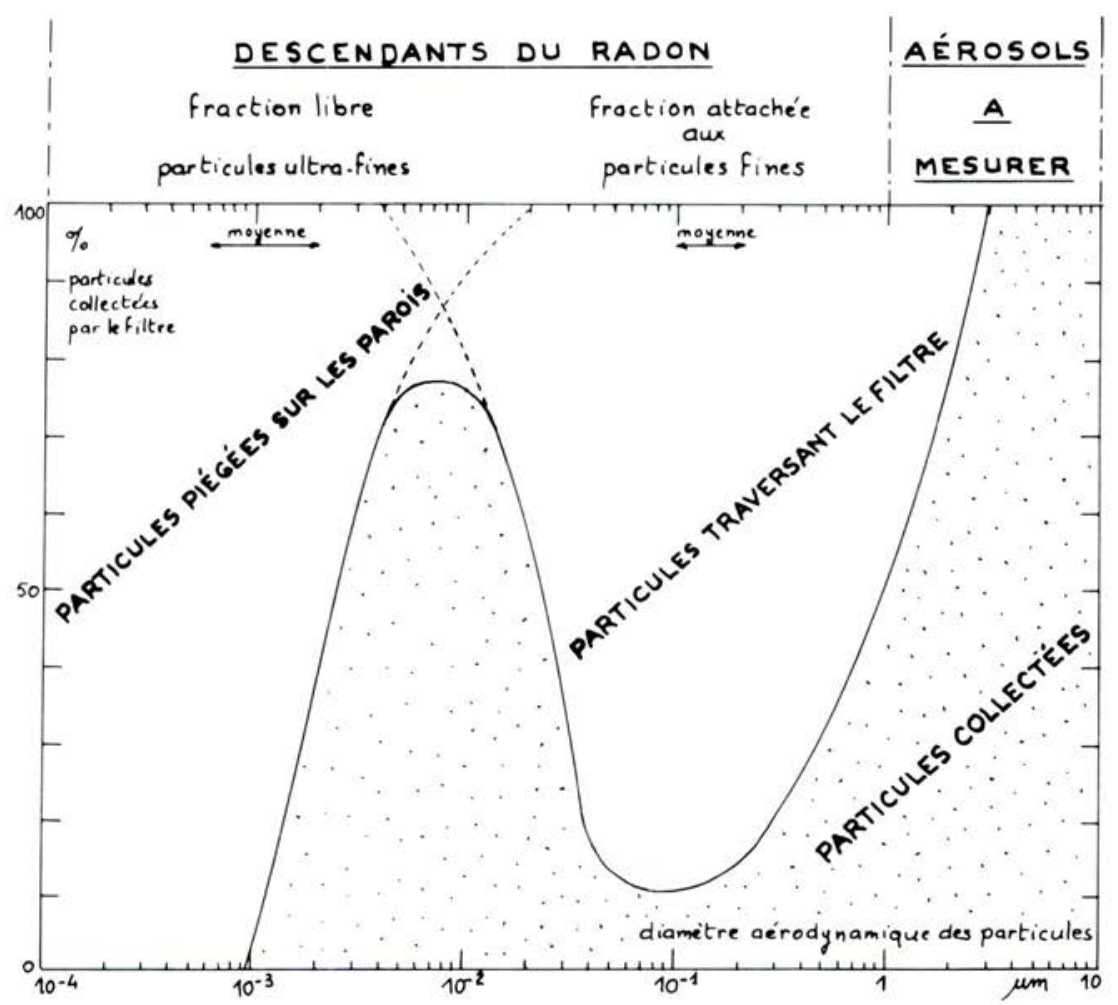

Fig. 2. - Séparation granulométrique effectuée par l'appareil.

L'expérimentation n'ayant pas été faite pour $d_{p}>0,1 \mu \mathrm{m}$, nous l'avons réalisée pour trois membranes ayant des diamètres de pores de $3-5$ et $8 \mu \mathrm{m}$. 


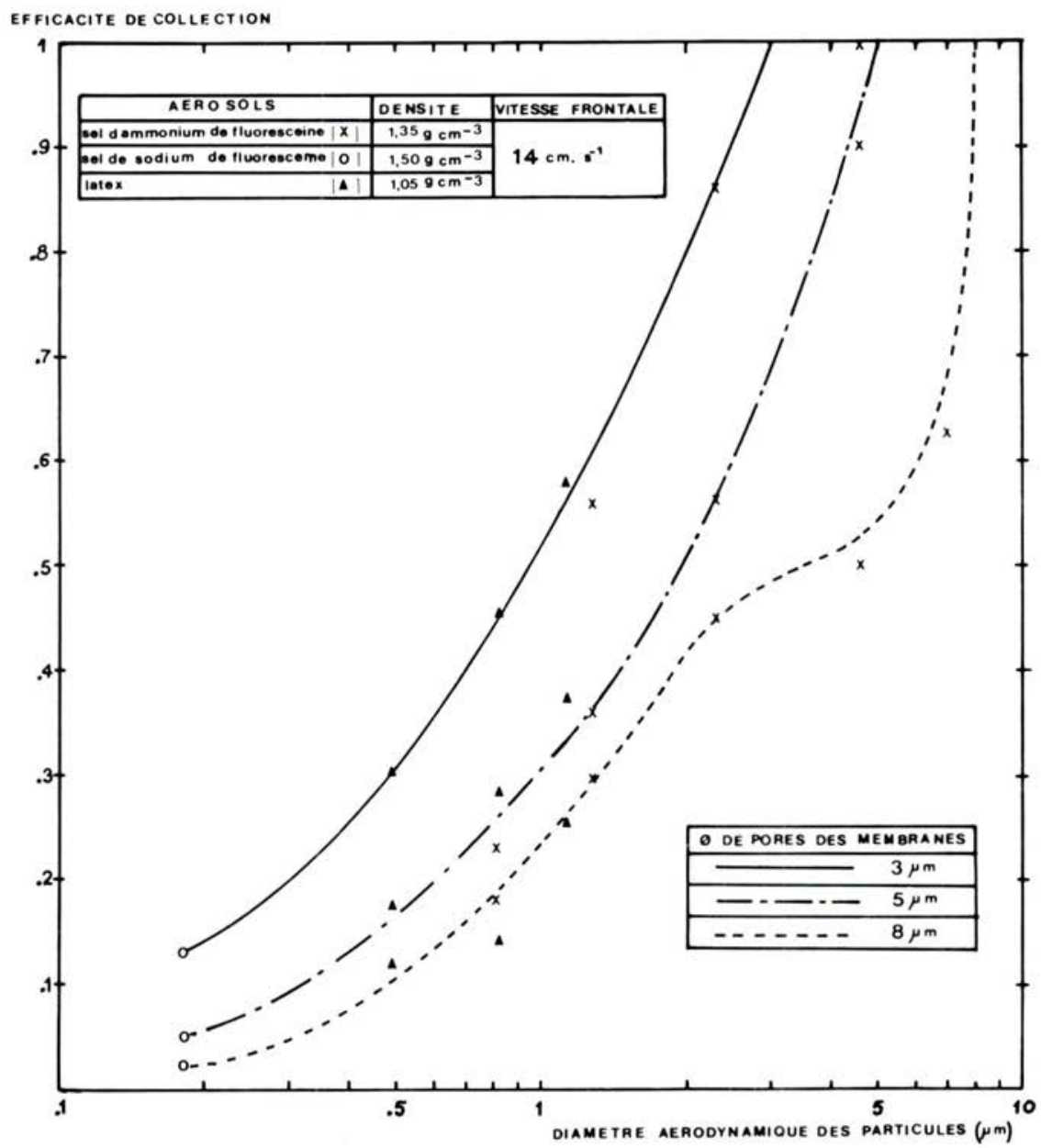

Fig. 3. - Courbes d'efficacité de collection de membranes Nuclépore.

\section{Expérimentation}

\subsection{Tête de prélèvement}

Les mesures de rendement de captation, de pertes de particules sur les parois, et d'homogénéité du prélèvement sur le filtre, sont réalisées à l'aide de particules monodispersées de $1,1 \mu \mathrm{m}-4,1 \mu \mathrm{m}$ et 9,0 $\mu \mathrm{m}$ pro- 
duites par un générateur à orifice vibrant [3]. II s'agit de sel d'ammonium de fluorescéine de masse volumique $1,35 \mathrm{~g} \cdot \mathrm{cm}^{-3}$; les diamètres aérodynamiques équivalents sont : $1,3-4,8-10,5 \mu \mathrm{m}$. Leur charge électrique est neutralisée par une source $\beta$ de krypton 85 . Les masses des particules collectées par le filtre ou déposées sur les parois sont recueillies par lavage et mesurées par un fluorimètre. L'appareil aspire les aérosols dans une chambre cylindrique $(\varnothing 13 \mathrm{~cm})$ parcourue par un écoulement à vitesse moyenne $0,13 \mathrm{~m} \cdot \mathrm{s}^{-1}$ parallèle au plan de l'orifice (tableau I).

3.1.1. Rendement de captation; il est voisin de $100 \%$. Remarquons que la valeur excessive obtenue pour $10,5 \mu \mathrm{m}(126 \%)$ est probablement due à un mode opératoire qui ne donne pas une mesure suffisamment précise de la concentration réelle $C_{0}$ des grosses particules. Ces essais devraient être confirmés en utilisant un tunnel de grande dimension qui permette d'effectuer simultanément le prélèvement par l'appareil et la mesure de $C_{0}$ par une sonde isocinétique à becs effilés.

3.1.2. Pertes sur les parois: elles sont faibles pour $1,3 \mu \mathrm{m}(1,3 \%)$ et $4,8 \mu \mathrm{m}(2,3 \%)$, et encore acceptables pour les plus grosses particules : $7,1 \%$ pour $10,5 \mu \mathrm{m}$.

3.1.3. Répartition du prélèvement sur le filtre : elle est déterminée en mesurant séparément les masses prélevées sur le filtre découpé en trois parties concentriques. On calcule la masse déposée par unité de surface de chacune de ces couronnes, et on la compare à la valeur moyenne sur le filtre entier. Leur rapport exprime le coefficient de répartition : plus il est voisin de 1 , plus le prélèvement est homogène. Pour 1,3 $\mu \mathrm{m}$ il est parfaitement homogène ; pour 4,8 et $10,5 \mu \mathrm{m}$, le prélèvement central est légèrement plus faible, au bénéfice de la couronne externe.

TABLEAU I

\begin{tabular}{|c|c|c|c|c|c|}
\hline Aérosol & \multicolumn{2}{|c|}{ Tête de prélèvement } & \multicolumn{3}{c|}{$\begin{array}{c}\text { Répartition des aérosols } \\
\text { sur le filtre }\end{array}$} \\
\hline $\begin{array}{c}\text { Diamètre } \\
\text { aérodynamique } \\
\mu \mathrm{m}\end{array}$ & $\begin{array}{c}\text { Rendement } \\
\text { de } \\
\text { captation } \%\end{array}$ & $\begin{array}{c}\text { Pertes sur } \\
\text { les parois } \\
\%\end{array}$ & $\begin{array}{c}\text { Couronne } \\
\text { externe }\end{array}$ & $\begin{array}{c}\text { Couronne } \\
\text { médiane }\end{array}$ & $\begin{array}{c}\text { Disque } \\
\text { central }\end{array}$ \\
\hline 1,3 & 99 & 1,3 & 0,98 & 1,08 & 0,96 \\
4,8 & 98 & 2,3 & 1,20 & 0,99 & 0,77 \\
10,5 & 126 & 7,1 & 1,28 & 0,84 & 0,76 \\
\hline
\end{tabular}

\subsection{Membranes Nuclépore}

3.2.1. Conditions d'essais: l'efficacité de collection des membranes Nuclépore de porosité 3 - 5 et $8 \mu \mathrm{m}$ est mesurée dans les conditions d'utilisation de l'appareil pour des particules de 0,1 à $6 \mu \mathrm{m}$.

L'empoussiérement moyen des laboratoires ventilés correspond à $30.10^{-6} \mathrm{~g} \cdot \mathrm{m}^{-3}$. L'appareil aspire donc en moyenne $5,4 \cdot 10^{-6} \mathrm{~g} \cdot \mathrm{h}^{-1}$ de poussières. Les essais sont réalisés de manière à capter des masses de quelque $10^{-6} \mathrm{~g}$ à quelque $10^{-5} \mathrm{~g}$. 
Des particules de diamètre moyen volumique de $0,15 \mu \mathrm{m}$ et de masse volumique de $1,5 \mathrm{~g} . \mathrm{cm}^{-3}$ sont produites par un générateur d'uranine (sel de sodium de fluorescéine). Des particules monodispersées de 0,7 - 1,1 $2,0-4,1$ et $6,1 \mu \mathrm{m}$ de sel d'ammonium de fluorescéine (masse volumique $1,35 \mathrm{~g} \mathrm{~cm}^{-3}$ ) sont obtenues à l'aide du générateur à orifice vibrant. Dans les deux cas, on mesure par fluorimétrie les masses recueillies sur la membrane $\left(m_{1}\right)$ et sur un filtre absolu situé en aval $\left(m_{2}\right)$. L'efficacité de collection est :

$$
E=\frac{m_{1}}{m_{1}+m_{2}}
$$

Des suspensions de sphères de latex parfaitement calibrées, de 0,48 0,81 et $1,10 \mu \mathrm{m}$ (masse volumique $1,05 \mathrm{~g}^{-\mathrm{cm}^{-3}}$ ) sont pulvérisées à l'aide d'une colonne Jouan, en verre. Les concentrations numériques des particules sont mesurées en amont $\left(n_{0}\right)$ et en aval $(n)$ de la membrane par un photomètre Royco. L'efficacité de collection est :

$$
E=\frac{n_{0}-n}{n_{0}}
$$

3.2.2. Résultats: Les pertes de charge sont relativement faibles: 3,5 mbar pour la porosité $8 \mu \mathrm{m}, 4$ mbar pour la porosité $3 \mu \mathrm{m}, 10$ mbar pour la porosité $5 \mu \mathrm{m}$, pour une vitesse frontale de $14 \mathrm{~cm} \cdot \mathrm{s}^{-1}$. Ces pertes de charge, de même que les efficacités de collection, évoluent peu avec la masse déposée lors des essais. Cela permet d'envisager l'utilisation continue de l'appareil pendant une journée de travail de $8 \mathrm{~h}$ sans modifier la qualité de la séparation granulométrique.

La figure 3 montre les courbes de l'efficacité de collection en fonction du diamètre aérodynamique des particules. La membrane de porosité $3 \mu \mathrm{m}$ répond le mieux à notre besoin puisque :

- le diamètre de coupure granulométrique est $1 \mu \mathrm{m}(E=52 \%)$;

- l'efficacité est élevée pour les grosses particules : $E=80 \%$ à $2 \mu \mathrm{m}$ et $100 \%$ au-dessus de $3 \mu \mathrm{m}$;

- l'efficacité est relativement faible pour l'aérosol radioactif naturel: $10 \%$ pour $0,1 \mu \mathrm{m}$ à $15 \%$ pour $0,2 \mu \mathrm{m}$.

Remarquons que la rupture de pente de la courbe d'efficacité de la membrane $8 \mu \mathrm{m}$ pour des particules supérieures à $3 \mu \mathrm{m}$ provient sans doute du rebond de ces particules possédant une inertie suffisante, ce qui leur donne une chance supplémentaire d'être entraînées à travers un pore.

\section{Conclusion}

L'expérimentation de la tête de prélèvement que nous avons conçue pour assurer la détection individuelle de la contamination atmosphérique alpha, répond aux exigences fixées. Aspirant, à l'aide d'une petite pompe, un débit de 3 I. $\mathrm{min}^{-1}$, cette tête :

- capte les aérosols jusqu'à $10 \mu \mathrm{m}$ avec un rendement voisin de $100 \%$;

- piège sur ses parois les particules ultrafines constituant la fraction libre du radon ; 
- conduit sans perte importante $(<7 \%)$ les particules les plus grosses $(10 \mu \mathrm{m})$ jusqu'au filtre de prélèvement sur lequel elles se répartissent de manière homogène ;

- sépare les particules submicroniques, qui traversent la membrane à pores capillaires, des grosses particules qui sont retenues ;

- mesure l'activité $\alpha$ de ces grosses particules par un semicteur dont la surface sensible, légèrement supérieure à celle du filtre, en est éloignée de $3 \mathrm{~mm}$.

La figure 2 montre la séparation granulométrique effectuée par l'appareil. Grâce à l'élimination de la quasi-totalité (98\% en théorie) de la fraction libre des descendants solides du radon, et d'environ $88 \%$ de ceux fixés sur les aérosols naturels, le bruit de fond dû au radon de la mesure de l'activité $\alpha$ devrait diminuer d'un facteur 10 à 20 environ.

Enfin, la petite taille de cette tête sphérique $(\varnothing=55 \mathrm{~mm})$, et le poids relativement faible du prototype réalisé en duralinox $(450 \mathrm{~g}$ avec la pompe et la batterie), permettent de réaliser un appareil individuel (cf. paragraphe II.2.1. et figure 1).

\section{CONCEPTION ET EXPERIMENTATION DE LA PARTIE DETECTION}

\section{Objectif}

II faut que la partie électronique de détection soit la plus petite possible afin de pouvoir être intégrée dans la tête de prélèvement.

\section{Conception}

L'ensemble de détection est constitué par une jonction à barrière de surface dont la partie sensible a un diamètre de $24 \mathrm{~mm}$. L'ensemble de mesure est constitué par un amplificateur de charge suivi d'un étage discriminateur-mise en forme. Les impulsions issues de cet étage commandent un haut parleur type "montre bracelet". Tout l'ensemble est alimenté sous une tension unique de $12 \mathrm{~V}$ "continu".

\section{Expérimentation}

\subsection{Rappels théoriques}

Dans le cas d'une mesure sur un filtre d'un corps à période longue, devant la durée de prélèvement l'on peut faire la représentation suivante (figure 4).

L'activité mesurée sur le filtre s'écrit $y=k . t$. En effet, nous savons que l'activité mesurée sur le filtre après un temps de prélèvement $t$ appelé tap, s'écrit :

$$
\begin{gathered}
A_{\text {(tap) }}\left(i \cdot s^{-1}\right)=3,7 \cdot 10^{10} \cdot C\left(C i \cdot m^{-3}\right) \cdot D\left(m^{3} \cdot s^{-1}\right) \cdot \operatorname{tap}(s) \text { ou } \\
=3,710^{10} \cdot C\left(C i \cdot m^{-3}\right) \cdot D\left(m^{3} \cdot h^{-1}\right) \cdot \operatorname{tap}(h)
\end{gathered}
$$

Si nous effectuons un comptage pendant un temps égal à $\theta$, nous aurons un nombre d'impulsions $\mathrm{N}$ qui sera :

$$
\mathrm{N}=\text { k.tap. } \theta
$$




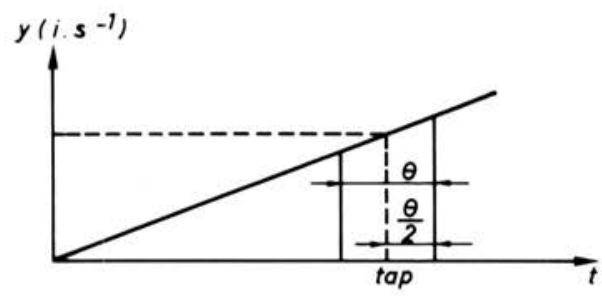

Fig. 4. - Activité déposée sur le filtre en fonction du temps. $\theta=$ temps de comptage.

3.2. Cas pratique du bruit de fond obtenu avec une concentration volumique de radon comprise entre $10^{-9}$ et $10^{-11} \mathrm{Ci}^{-3}$

Compte tenu de l'étude aéraulique de la tête de prélèvement et du filtre choisi, nous devrions obtenir des taux de comptage dus aux descendants du radon très faibles. Les essais du moniteur individuel sont réalisés par le Service de protection des rayonnements du CEN/Saclay à l'aide d'émissions de radon faites dans un laboratoire non ventilé avec les concentrations comprises entre $10^{-10}$ et $10^{-9} \mathrm{Ci} . \mathrm{m}^{-3}$.

Les premiers résultats sont résumés dans le tableau II.

TABLEAU ॥

\begin{tabular}{|c|c|c|c|}
\hline \multirow{2}{*}{$\begin{array}{c}\text { Concentration radon } \\
\text { en Ci.m }\end{array}$} & \multicolumn{2}{|c|}{ Taux de comptage } & \multirow{2}{*}{$\begin{array}{c}\text { Coefficient d'élimination } \\
\text { du radon }\end{array}$} \\
\cline { 2 - 3 } & $\mathrm{i} / \mathrm{s}$ & $\mathrm{i} / 10 \mathrm{~s}$ & 11,4 \\
\hline $8 \cdot 10^{-10}$ & 0,178 & 1,78 & 9 \\
\hline $4 \cdot 10^{-10}$ & 0,113 & 1,113 & 8,2 \\
\hline $1.10^{-10}$ & 0,031 & 0,31 & 13 \\
\hline $1.10^{\cdot 9}$ & 0,195 & 1,95 & 13 \\
\hline
\end{tabular}

Les résultats obtenus pratiquement confirment dans l'ensemble les résultats théoriques attendus; en effet, $90 \%$ des descendants solides du radon sont éliminés puisque le facteur de compensation est voisin de 10 .

Pour une valeur de $10^{-10} \mathrm{Ci} \cdot \mathrm{m}^{-3}$ nous obtenons de l'ordre de 1 impulsion pour $30 \mathrm{~s}$. Compte tenu de ce bruit de fond, une étude mathématique du seuil de détection $[2,8]$ a montré qu'il était possible de détecter 25 CMA.h $\mathrm{de}^{239} \mathrm{Pu}$.

\section{Conclusions}

Les résultats obtenus sont très encourageants. En effet, il devient possible de détecter en continu de faibles concentrations de ${ }^{239} \mathrm{Pu}$ sans système électronique sophistiqué de compensation radon qui nécessite un volume de matériel plus important. 
L'on peut estimer une sensibilité pratique $\simeq 25 \mathrm{CMA}$.h et ceci est à comparer avec la sensibilité du CFSA (balise $\mathrm{Pu}$ ) mise au point au CEA qui est de $\simeq 2$ à 5 CMA.h.

L'on peut dire qu'en cas d'incident de contamination occasionné par l'opérateur, donc à sa proximité immédiate, ce qui est le cas le plus probable, le moniteur individuel peut immédiatement aspirer comme le nez de l'opérateur une concentration très nettement supérieure (10 à 20 fois plus) à celle qu'aspirera la balise de zone avec un certain retard (une à quelques minutes).

Ainsi, malgré une sensibilité de l'ordre de 5 à 10 fois moins bonne que celle de la balise de zone mais compte tenu des paramètres de transferts (coefficient et vitesse) qui jouent en faveur du moniteur individuel, cet appareil peut donner une alarme plus rapide et une mesure plus représentative de la contamination individuelle. Ce procédé, qui mérite, sans doute, un développement industriel, a donné lieu à une demande de brevet [9].

\section{BIBLIOGRAPHIE}

[1] BOULAUD D., CHOUARD J.C., MADELAINE G. Contribution à l'étude de l'efficacité des membranes filtrantes. Communication à Filtra 80, Paris 1517 octobre 1980.

[2] CEA. Comité d'Instrumentation de radioprotection (CIR). Seuils et limites de détection - Application aux mesures de radioactivité par comptage Application aux appareils de radioprotection. (CIR-GTTA - GTR3 $n^{\circ} 7$ ). Fontenayaux-Roses : CEA - CIR, 1983.

[3] CHARUAU J. Optimisation de la détection des aérosols radioactifs dans un local. In: La ventilation des installations, Pierrelatte, 22-26 mars 1982. Fontenay-auxRoses: SFRP, 1982.

[4] CHARUAU J. Etude du dépôt des particules dans les conduits: Optimisation des tubes de prélèvement des aérosols radioactifs. Thèse, Paris VI, 1981 et rapport CEA R-5158 (1982).

[5] DAVIES C.N. The sampling of aerosols - The entry of aerosols into sampling tubes and heads. Staub, 1968, 28, n० 6 .

[6] DUPORT P. L'aérage et les caractéristiques de l'atmosphère d'une mine d'uranium laboratoire. Application à la répartition des particules dans les voies respiratoires. Thèse d'Université, Brest, 1976 et rapport CEA R-4927, 1978.

[7] TYMEN G. Données actuelles sur la granulométrie et l'état de charge des aérosols porteurs des descendants du radon. Séminaire sur la radioprotection dans les mines d'uranium, Limoges, 5-6 octobre 1982.

[8] LASSEUR $\mathrm{Cl}$. Le dosage des radionucléides par la méthode des prélèvements successifs de courte durée. Application à la mesure de la concentration et du déséquilibre des descendants du radon dans les atmosphères libres et conditionnées. Thèse d'Université, Toulouse et rapport CEA R-4048, 1970.

[9] BuRghoffer P., ChARUAu J., MERELLI M., PRIGENT R. Procédé de détection de la contamination atmosphérique par des aérosols de particules alpha et détecteur portatif de cette contamination mettant en œuvre le procédé. Brevet français $n^{\circ} 8309685,10$ juin 1983. 